\title{
Precision Fabrication of a Large-Area Sinusoidal Surface Using a Fast-Tool-Servo Technique -Improvement of Local Fabrication Accuracy_*
}

\author{
Wei GAO**, Makoto TANO**, Takeshi ARAKI*** and Satoshi KIYONO**
}

This paper describes a diamond turning fabrication system for a sinusoidal grid surface. The wavelength and amplitude of the sinusoidal wave in each direction are $100 \mu \mathrm{m}$ and $100 \mathrm{~nm}$, respectively. The fabrication system, which is based on a fast-tool-servo (FTS), has the ability to generate the angle grid surface over an area of $\phi 150 \mathrm{~mm}$. This paper focuses on the improvement of the local fabrication accuracy. The areas considered are each approximately $1 \times 1 \mathrm{~mm}$, and can be imaged by an interference microscope. Specific fabrication errors of the manufacturing process, caused by the round nose geometry of the diamond cutting tool and the data digitization, are successfully identified by Discrete Fourier Transform of the microscope images. Compensation processes are carried out to reduce the errors. As a result, the fabrication errors in local areas of the angle grid surface are reduced by $1 / 10$.

Key Words: Measurement, Accuracy, Ultra-Precision Machining, Sinusoidal Surface, Fast-Tool-Servo

\section{Introduction}

The authors have proposed a surface encoder for multi-degree-of-freedom position detection ${ }^{(1),(2)}$. The surface encoder has two elements: an angle grid, which is the superposition of sinusoidal waves in the $X$ - and $Y$ directions (see Fig. 1), and a two-dimensional slope sensor for reading local slope profiles of the grid surface along the $X$ - and $Y$-axes. The height profile can be expressed via Eq. (1):

$$
f(x, y)=a \sin \left(\frac{2 \pi}{\lambda} x\right)+a \sin \left(\frac{2 \pi}{\lambda} y\right)
$$

where $a$ is the amplitude and $\lambda$ is the spatial wavelength. The angle grid surface has a spatial wavelength of $100 \mu \mathrm{m}$ and amplitude of $100 \mathrm{~nm}$ in both of the $X$ - and $Y$-directions. Since the angle grid surface is used as the reference for position measurement, it is necessary to fabricate the sinusoidal surface with a high accuracy over a

${ }^{*}$ Received 14th July, 2006 (No. 05-0318). Japanese Original: Trans. Jpn. Soc. Mech. Eng., Vol.71, No.712, C (2005), pp.3602-3607 (Received 22nd March, 2005)

** Department of Nanomechanics, Tohoku University, 6-601 Aramaki Aza Aoba, Aoba-ku, Sendai-shi, Miyagi 9808579, Japan. E-mail: gaowei@cc.mech.tohoku.ac.jp

*** Hitachi Displays Ltd., AKS Bldg. 5F, 6-2 Kanda Neribeicho 3, Chiyoda-ku, Tokyo 101-0022, Japan large area.

The authors have constructed a fabrication system for the angle grid surface based on single-point diamond turning by employing a fast-tool-servo technique ${ }^{(3),(4)}$. Diamond turning has the advantage of a good surface finish. Using a fast-tool-servo technique, this system yields high form accuracy. The system has enabled the fabrication of a sinusoidal surface over $\phi 150 \mathrm{~mm}^{(5)}$.

A surface encoder is required for an accuracy of $100 \mathrm{~nm}$ and a resolution of $10 \mathrm{~nm}$. For this, the profile accuracy must be at the nanometer level in the height direction $(Z)$ and sub-micrometer level in the $X$ - and $Y$ directions. The present paper introduces an evaluation method that can efficiently identify error components of the fabricated surface. The improvement in fabrication

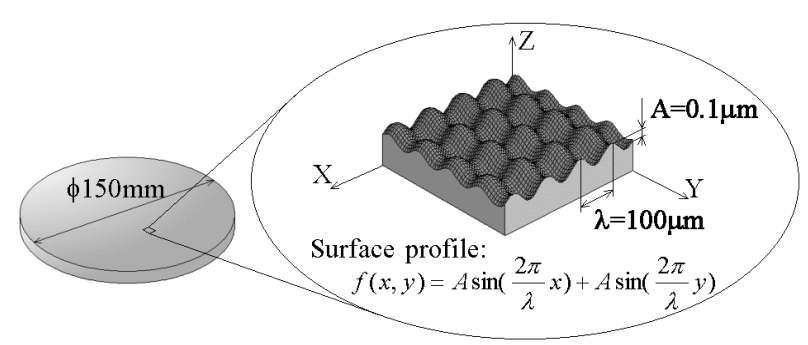

Fig. 1 Schematic of the angle grid 
accuracy achieved through feeding back the evaluation results to the fabrication process is reported.

\section{Fabrication System}

Figure 2 schematizes the fabrication system for the angle grid. The system is composed of an ultra-precision lathe, a fast-tool-servo (FTS) and a personal computer. The FTS was mounted on the X-slide of the ultra-precision lathe, and the workpiece was held on the spindle with its axis along the $Z$-direction by a vacuum chuck. Because the motion of the X-slide and the rotation of the spindle are synchronized, it is enough to get the rotary encoder pulse of the spindle in order to obtain the position of the tool and the workpiece. The polar coordinates of the tool tip position in the $X Y$ plane is given by Eq. (2):

$$
\left(r_{i}, \theta_{i}\right)=\left(r_{0}-\frac{F i}{P T}, 2 \pi \frac{i}{P}\right) \quad i=0,1, \ldots, N-1
$$

where $r_{0}$ is the radius of workpiece in $\mathrm{mm}, F$ is the feed rate of the $\mathrm{X}$-slide in $\mathrm{mm} / \mathrm{min}, P$ is the pulse number of the rotary encoder of the spindle in each revolution in units of pulse/revolution, $T$ is the rotational speed of the spindle in units of revolution/min, $i$ is the $i$-th rotary encoder pulse, and $N$ is the total pulse number of the rotary encoder for the $\mathrm{X}$-slide reaching the center. Along the spiral trajectory, the depth of cut of the diamond tool along the $Z$ direction is controlled by an FTS. When fabrication starts, the cutting data are output to the controller one by one responding to the trigger signal from the rotary encoder of the spindle. From Eqs. (1) and (2), the depth of cut at the $i$-th rotary encoder pulse can be obtained:

$$
\begin{aligned}
z(i) & =f\left(r_{i} \cos \theta_{i}, r_{i} \sin \theta_{i}\right) \\
& =f\left(\left(r_{0}-\frac{F i}{P T}\right) \cos \left(2 \pi \frac{i}{P}\right),\left(r_{0}-\frac{F i}{P T}\right) \sin \left(2 \pi \frac{i}{P}\right)\right)
\end{aligned}
$$

The depth of cut at each point is calculated as follows and stored in a personal computer before fabrication. In the first fabrication, the workpiece material is A5052, the

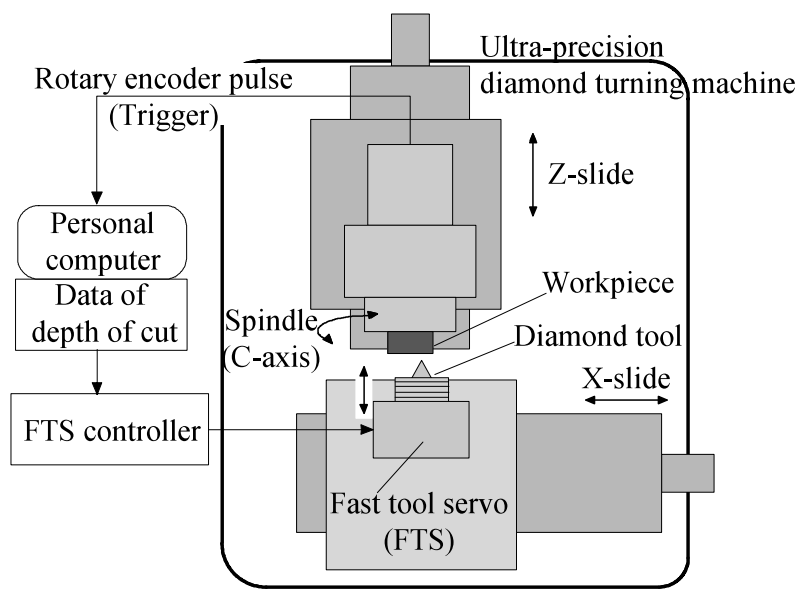

Fig. 2 Fabrication system for the angle grid rotary encoder pulse in each revolution is 30000 , the rotational speed of the spindle is $20 \mathrm{rpm}$, the feed rate of the $\mathrm{X}$-slide is $5 \mu \mathrm{m} / \mathrm{rev}$ and the diameter of the workpiece is $150 \mathrm{~mm}$.

\section{Evaluation Method}

In consideration of the profile of the angle grid, an interference microscope is chosen as profiling instrument. However, this microscope has a measurement area of approximately $1 \times 1 \mathrm{~mm}$. The measurement of the whole area is not effective. On the other hand, from the point of view of the turning process shown in Fig. 3, characteristics of the profile errors of regions A, B, C ... along the circumference direction should be similar, and the evaluation results of one of these regions is representative of the others as well. Thus, we chose several regions along the radial direction, which were numbered $0,1,2 \ldots 7$ in Fig. 3, as representative regions for evaluating profile errors. The evaluation region numbered 0 is in the workpiece center.

Because the angle grid has a sinusoidal profile, the Fourier transform is expected to be an effective evaluation method. Thus, the two-dimensional discrete Fourier transform (2D DFT) of the interference microscope image is employed for identification of error components in the surface profile. Figures 4 and 5 are an interference microscope image and the spectrum obtained by the 2D DFT in area 7, respectively. In Fig. 5, the $f_{X}$ and $f_{Y}$ axes show spatial frequency in the $X$ - and $Y$-directions, respectively. The $m\left(f_{X}, f_{Y}\right)$ axis shows the amplitude of the spec-

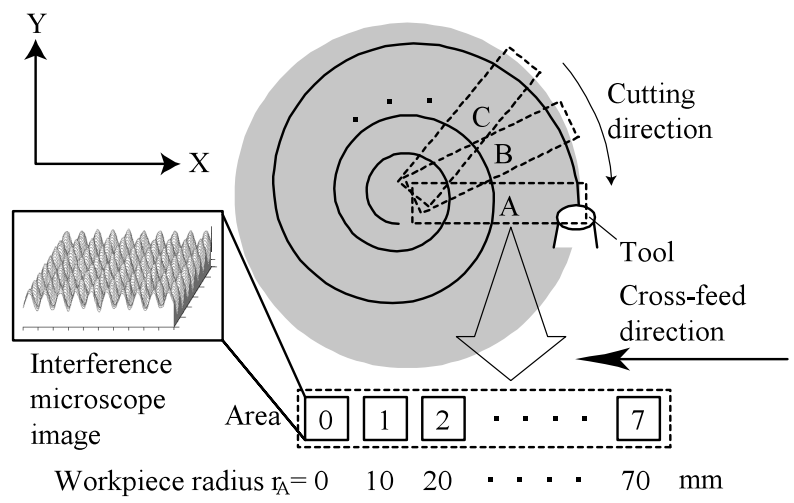

Fig. 3 Evaluation areas

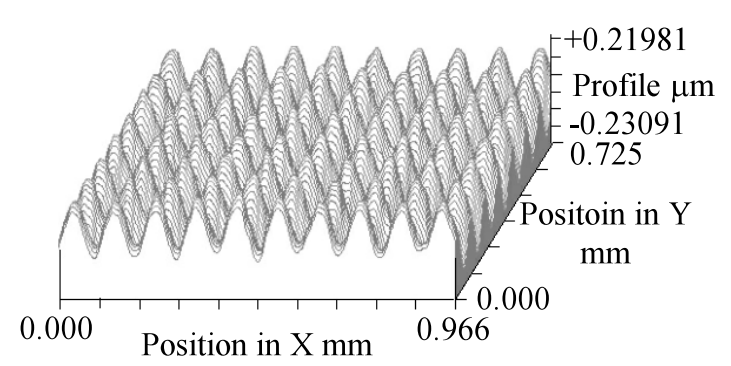

Fig. 4 Interference microscope image of the angle grid in Area 7 

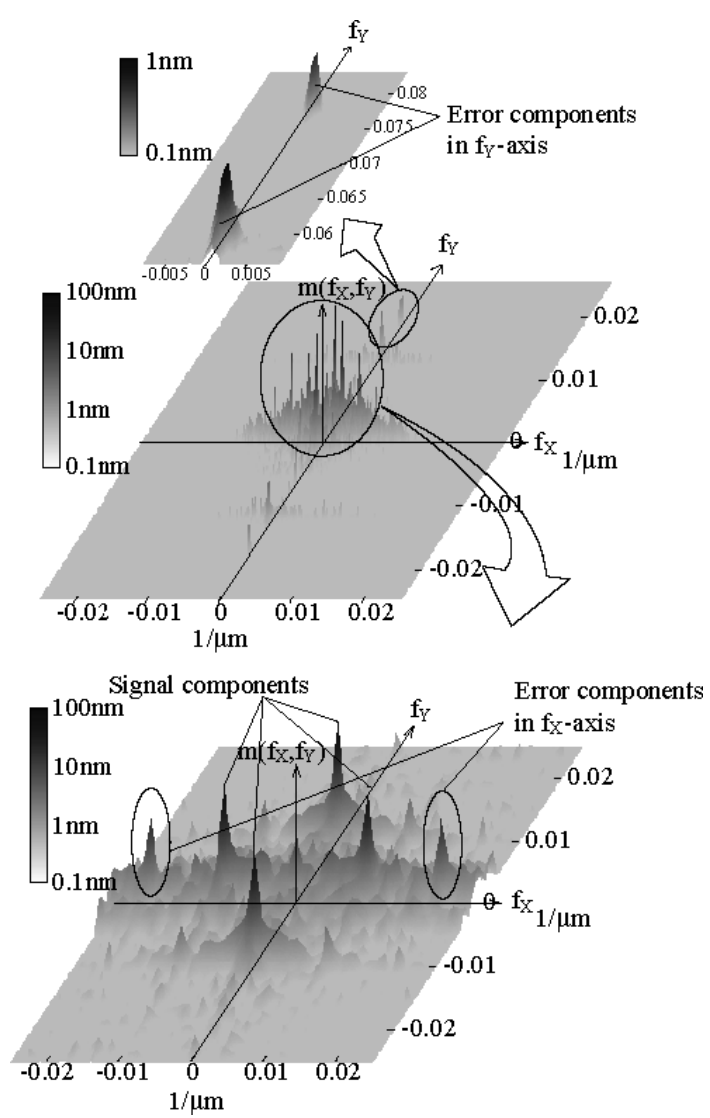

Fig. 5 Spectrum distribution of the surface profile in Fig. 4

trum. The four largest components at the frequency of $0.01 \mu^{-1}$, which corresponds to a wavelength of $100 \mu \mathrm{m}$, show the desired sinusoidal surface profile. Other components shown in the spectrum are profile errors. These components provide information on not only the spatial frequency but also directions of profile errors. On the basis of the interference microscopy specification, the minimum and maximum spatial wavelengths are $4.6 \mu \mathrm{m}$ and $1 \mathrm{~mm}$, respectively.

\section{Compensation of Errors}

\subsection{Error caused by the round nose geometry}

As can be seen in Fig. 5, the spectrum has error peaks at $0.02 \mu \mathrm{m}^{-1}$ in the $f_{X}$ axis, which is half of the spatial wavelength of the angle grid. These error peaks can be seen in all areas. The sizes of the error peaks pertaining to each area are tabulated in Table 1. Error peaks typically occur only in the tool feed direction and at half the spatial frequency of the profile component. When the cutting data are calculated as intact profile of the angle grid, the cutting points are different from the programmed cutting position, as shown in Fig. 6. This difference causes the profile error. Because the differential value is zero at the top and bottom of the sinusoidal wave, the profile shows no errors and the wavelength of profile errors becomes equal to half the angle grid wavelength. A simulation of this error is
Table 1 Errors caused by the round nose geometry

\begin{tabular}{|c|c|c|c|c|c|c|c|}
\hline Evaluation area & 1 & 2 & 3 & 4 & 5 & 6 & 7 \\
\hline $\begin{array}{c}\text { Workpiece } \\
\text { radius } \mathrm{r}_{\mathrm{A}}[\mathrm{mm}]\end{array}$ & 10 & 20 & 30 & 40 & 50 & 60 & 70 \\
\hline Error [nm] & 7.8 & 9.5 & 9.5 & 10.4 & 10.2 & 11.5 & 13.2 \\
\hline
\end{tabular}

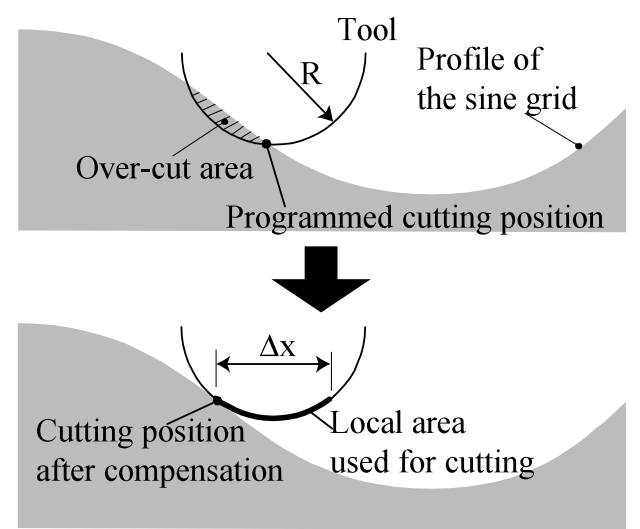

Fig. 6 Compensation of the profile error caused by the round nose geometry

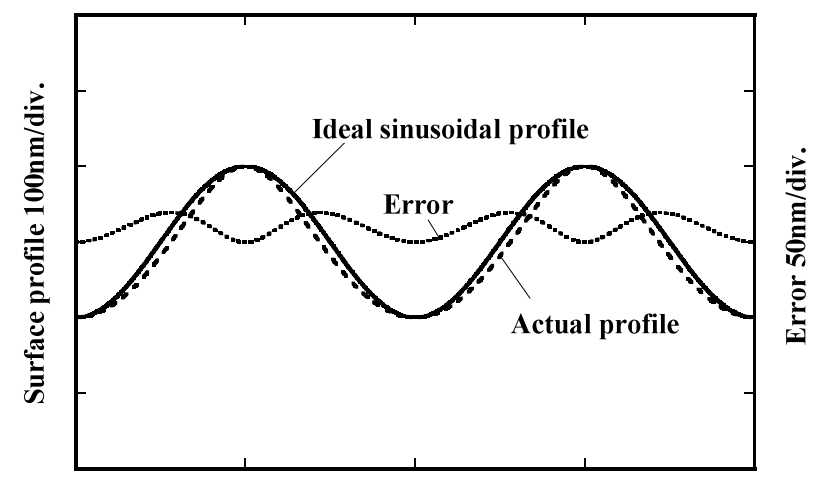

Position in X 50 $\mu \mathrm{m} / \mathrm{div}$.

Fig. 7 Simulation results of the profile error caused by the tool round nose geometry

performed. The results are presented in Fig. 7. The results show that the profile error, which has a wavelength of $50 \mu \mathrm{m}$, is caused by the difference in cutting points due to the round nose geometry.

The average value of the error peaks in a fabricated profile is $10.3 \mathrm{~nm}$, versus the $9.3 \mathrm{~nm}$ obtained by simulation. This error is caused by the difference between the ideal round nose radius $R$ and the actual tool radius $R^{\prime}$, which is used for cutting the angle grid. $\Delta x$ in Fig. 6 is the width of the tool edge used to cut the angle grid and is calculated by Eq. (4):

$$
\Delta x=\frac{2 R}{\sqrt{1+\left(\frac{\lambda}{2 \pi a}\right)^{2}}}
$$

where $R$ is the tool radius, and $\lambda$ and $a$ are the wavelength and amplitude of the angle grid, respectively. In the fabrication performed in the present study, $R=1000 \mu \mathrm{m}$, $\lambda=100 \mu \mathrm{m}$, and $a=0.1 \mu \mathrm{m}$. Thus, $\Delta x$ is calculated to be 


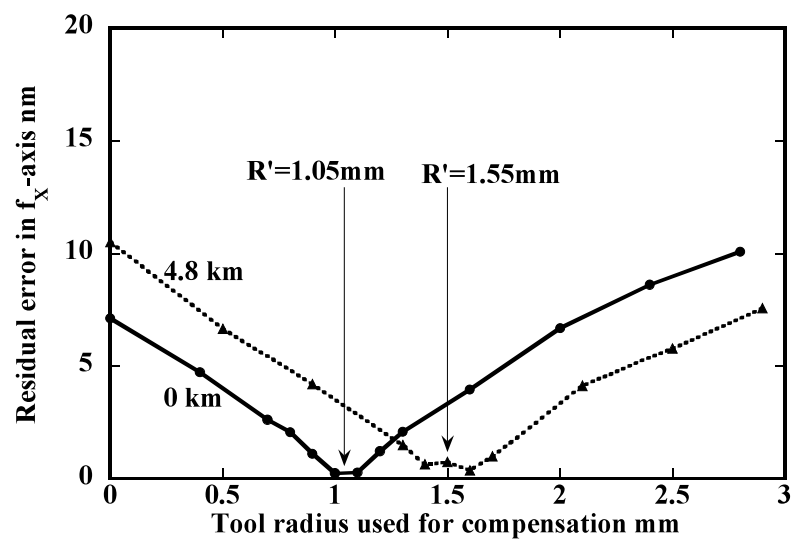

Fig. 8 Determination of the local radius used for compensation

$13 \mu \mathrm{m}$. This value is very small compared to the tool radius. The actual tool radius $R^{\prime}$ is thought to be different from the ideal round nose radius $R$.

To determine the actual tool radius $R^{\prime}$, angle grids are fabricated on the basis of the data, which is compensated by a certain tool radius value near the ideal round nose radius $R . \quad R^{\prime}$ is determined by $2 \mathrm{D}$ DFT analysis of the fabricated angle grid. The error caused by the difference between $R$ and $R^{\prime}$ can be reduced by compensated fabrication using $R^{\prime}$. At larger values of the workpiece radius, $f_{X}$ and $f_{Y}$ are assumed to be the feed and cutting directions, respectively. However, this assumption does not hold at the center of the workpiece, and hence the error due to the round nose geometry is distributed in both directions. Thus, the 2D DFT analysis is carried out near the outer edge of the workpiece.

Figure 8 shows the results obtained for the actual tool radius $R^{\prime}$. In addition, the results after $4.8 \mathrm{~km}$ cutting are shown to help elucidate the influence of tool detrition. A cutting length of $3.5 \mathrm{~km}$ is used in fabricating the angle grid. $R^{\prime}=1.85 \mathrm{~mm}$ on the basis of the preliminary fabrication, and the cutting length of the angle grid is used for compensated fabrication of the angle grid.

\subsection{Error caused by the digitization of cutting points}

Figure 5 shows the error peaks in the $f_{Y}$ axis. Figure 9 shows the size and spatial frequency of error peaks in each region. In Fig. 9, the outer area of the workpiece, the larger error peaks and the smaller spatial frequency are shown. In the circumferential direction $f_{Y}$ of the workpiece, the interval is proportional to the radius and inversely proportional to the number of rotary encoder pulses of the spindle per revolution. When the rotary encoder pulse is the same for all radii, the larger the radius is, the larger the interval is. Thus, the errors are thought to be caused by the digitization of cutting points, which results in an interval between adjacent cutting points.

The errors are simulated. The fast-tool-servo is modeled as a first-order lag element of $2.3 \mathrm{kHz}$, which is mea-

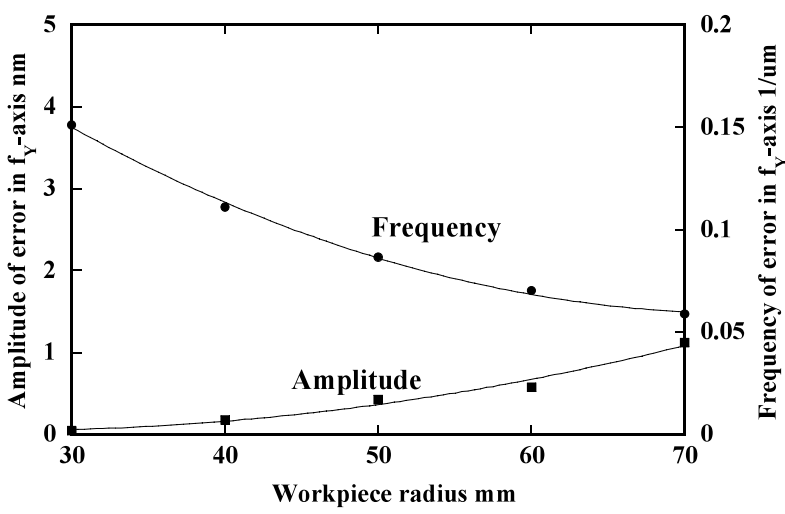

Fig. 9 Measurement results of the error caused by digitization

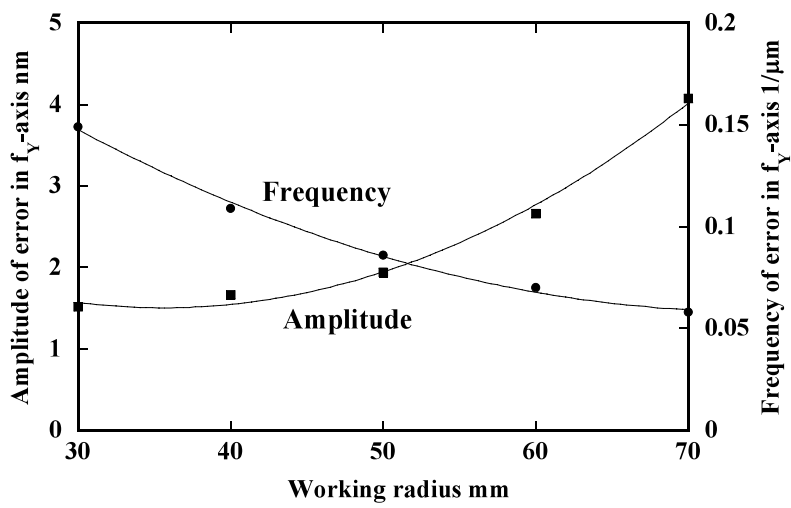

Fig. 10 Simulation results of the error caused by digitization

sured to be the cutoff frequency. Figure 10 shows the simulation results. It can be seen that the error peaks differ in size between the measurement and simulation results. However, the behavior revealed by the simulation is almost the same as that observed in the measurement results.

The error caused by the digitization of cutting points can be simply compensated by increasing the rotary encoder pulses per revolution and reducing the cutting point interval. On the other hand, the output of the cutting data from the digital-to-analog converter is supposed to cause a delay due to the limitation of the FTS frequency band. This problem is solved by reducing the rotation speed of the spindle, but the fabrication time would increase as a result. Thus, the fabrication conditions are modified.

The parameters are as follows:

$T$ : rotational speed of the spindle [revolution/min]

$P$ : pulse number of rotary encoder of spindle per revolution [pulse/revolution]

$t_{P}$ : track pitch in $X$ direction [ $\mu \mathrm{m} /$ revolution]

$r$ : radial position in workpiece $[\mu \mathrm{m}]$

Other parameters are calculated as follows:

$V=2 \pi r T$ : cutting speed $[\mu \mathrm{m} / \mathrm{min}]$

$F_{S}=\frac{P T}{60}:$ output frequency of cutting data $[\mathrm{Hz}]$

$L_{S}=\frac{2 \pi r}{P}:$ interval of the circumferential direction $[\mu \mathrm{m}]$

$F_{F T S}=\frac{2 \pi r T}{60 \lambda}:$ drive frequency of Fast-Tool-Servo $[\mathrm{Hz}]$ 


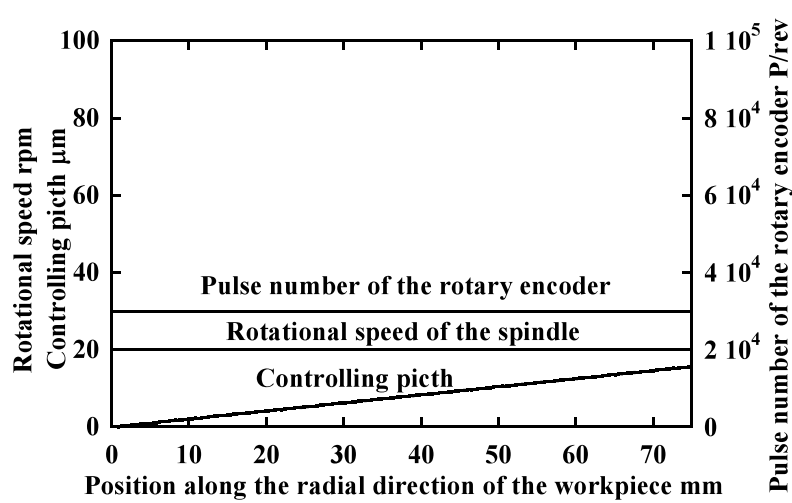

Fig. 11 Fabrication conditions before modification

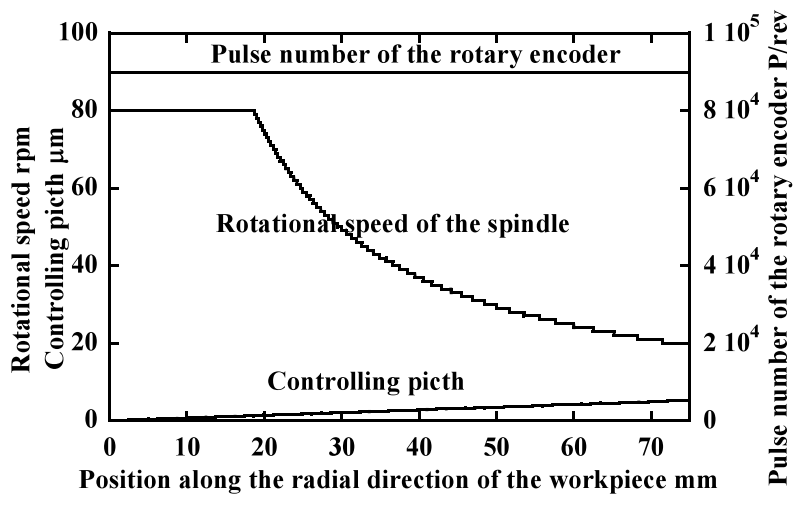

Fig. 12 Fabrication conditions after modification

$D_{P}=\frac{P r_{\mathrm{max}}}{t_{P}}:$ total number of cutting data points

The main constraints are as follows:

$T<167$ : response limit of the rotary encoder of the spindle $P<180$ 000: maximum number of rotary encoder pulses per revolution

$F_{F T S}<1$ 600: dynamic response of fast-tool-servo

Figure 11 shows the fabrication conditions before modification. The interval in the circumferential direction $L_{S}$ is $15.7 \mu \mathrm{m}$, which is one-sixth the wavelength of the angle grid. If the number of rotary encoder pulses per revolution increases by three times, $L_{S}$ becomes approximately $5 \mu \mathrm{m}$, which is the same as the track pitch $t_{P}$. However, the output frequency of the cutting data increases. The fabrication conditions are modified after investigation of the maximum output frequency in the fabrication system. An output error test of the fabrication system is carried out. Output conditions under different output frequencies are investigated using rectangular data like $0,1,0,1,0 \ldots$ as cutting data. Exceeding the output frequency limit leads to an absence of data like $0,1,0,0,1 \ldots$ This is detected by the glitch trigger function of the oscilloscope. No absence of data was observed for $10 \mathrm{~min}$ at less than $120 \mathrm{kHz}$.

Figure 12 shows the new fabrication conditions. $P$ is set to 90000 , which is three times larger than the corresponding value used before modification. The interval of

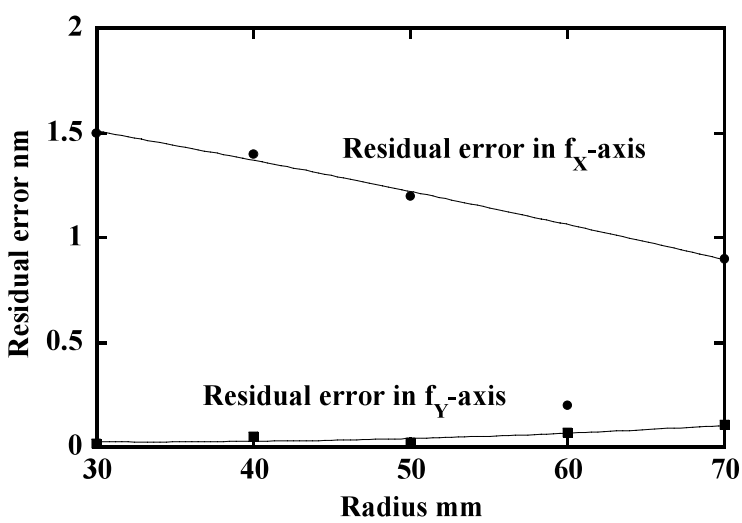

Fig. 13 Analysis results with modified fabrication conditions

cutting data in the circumferential direction at the outermost area is $5.2 \mu \mathrm{m}$, which can divide the wavelength of an angle grid into 20 . The rotational speed of the spindle is set to $T=1.5 \times 10^{6} / r \mathrm{rpm}[r$ is radius $](20-80 \mathrm{rpm})$ in regions farther than $20 \mathrm{~mm}$ from the center. As a result, the fabrication time is reduced to $6.6 \mathrm{~h}$, which is half the fabrication time required before modification of fabrication conditions. Within $20 \mathrm{~mm}$ of the center of the workpiece, the rotational speed of the spindle is constant at $80 \mathrm{rpm}$, which is limited by the maximum output frequency of $120 \mathrm{kHz}$.

\subsection{Compensated fabrication under new fabrica- tion conditions}

Compensated fabrication is performed after modification of fabrication conditions. A 2D DFT analysis of the angle grid fabricated is carried out. Figure 13 shows the size of the error caused by the round nose geometry of the cutting tool and the size of the error caused by digitization of cutting points. As can be seen, the former error is reduced from $13.2 \mathrm{~nm}$ to $1.5 \mathrm{~nm}$, while the latter error is reduced from $1 \mathrm{~nm}$ to $0.1 \mathrm{~nm}$.

\section{Conclusion}

Fabrication experiments are carried out using a fabrication system for an angle grid composed of a Fast-ToolServo and ultra-precision lathe. 2D DFT is employed to estimate the angle grid profile locally. As a result of the 2D DFT analysis, two error factors are identified: the round nose geometry of the cutting tool and the digitization of cutting data. These errors are reduced by a factor of 10 through investigation of the actual tool radius $R^{\prime}$ and modification of the fabrication conditions, respectively.

Future work includes estimation of global area and improvement of flatness. For example, measurement and compensation of the $\mathrm{X}$-slide motion error are necessary.

\section{Acknowledgement}

This work was supported by a grant-in-aid from JSPS (No. 14102017). 


\section{References}

( 1 ) Kiyono, S., Cai, P. and Gao, W., An Angle-Based Position Detection Method for Precision Machines, JSME Int. J., Ser. C, Vol.42, No.1 (1999), pp.44-48.

( 2 ) Gao, W., Dejima, S., Shimizu, Y. and Kiyono, S., Precision Measurement of Two-Axis Positions and Tilt Motions Using a Surface Encoder, Annals of the CIRP, Vol.52, No.1 (2003), pp.435-438.

( 3 ) Patterson, S.R. and Magrab, E.B., Design and Testing of a Fast Tool Servo for Diamond Turning, Precision Engineering, Vol.7, No.3 (1985), pp.123-128.

( 4 ) Okazaki, Y., Fast Tool Servo System and Its Application to Three Dimensional Fine Figures, Proc. of 13th ASPE, (1998), pp.100-103.

( 5 ) Gao, W., Araki, T., Kiyono, S., Okazaki, Y. and Yamanaka, M., Precision Nano-Fabrication and Evaluation of a Large Area Sinusoidal Grid Surface for a Surface Encoder, Precision Engineering, Vol.27, No.3 (2003), pp.289-298. 